\title{
Distribution of dipeptidyl-peptidase IV on keratinocytes in the margin zone of a psoriatic lesion: a comparison with hyperproliferation and aberrant differentiation markers
}

\author{
R. G. van Lingen · M. K. P. Poll • M. M. B. Seyger • \\ E. M. G. J. de Jong • P. C. M. van de Kerkhof • \\ P. E. J. van Erp
}

Received: 15 January 2008 / Revised: 3 April 2008 / Accepted: 28 April 2008 / Published online: 22 May 2008

(C) The Author(s) 2008

\begin{abstract}
The inflammation process in psoriatic skin is characterized by influx of leukocytes, hyperproliferation and aberrant differentiation of keratinocytes regulated via cytokines. Dipeptidyl-peptidase IV (DPPIV) is known to be upregulated on keratinocytes in the psoriatic lesion. The objective was to gain insight into dynamics of DPPIV expression and enzyme activity together with keratinocyte proliferation and differentiation markers during development of a psoriatic lesion, in order to investigate coherence in mechanisms behind the upregulation of DPPIV in psoriatic skin. The expression of DPPIV, Ki-67 antigen and keratin-16 (K16) was studied in the dynamic margin zone of the psoriatic lesion, examining skin sections of the clinically uninvolved skin, the early lesion and the chronic lesion of psoriatic patients compared to healthy volunteers using immunohistochemical and enzymehistochemical staining methods. DPPIV-expression and enzyme activity, Ki-67 antigen and K16 are significantly upregulated in the centre and inner margin of the lesion compared to clinically uninvolved skin and the healthy volunteers skin. Mutually between the centre and inner margin, this upregulation did not differ significantly. The clinical symptomless skin proved to have significantly elevated DPPIV enzyme activity compared to the skin of healthy volunteers. We demonstrate that DPPIV is expressed and enzymatically active well before the development of an overt psoriatic lesion. The abnormal DPPIV distribution in psoriatic skin does not coincide with known markers of aberrant growth and differ-
\end{abstract}

R. G. van Lingen $(\bowtie) \cdot$ M. K. P. Poll · M. M. B. Seyger

E. M. G. J. de Jong · P. C. M. van de Kerkhof · P. E. J. van Erp Department of Dermatology,

Radboud University Nijmegen Medical Centre, P.O. Box 9101, 6500 HB Nijmegen, The Netherlands e-mail: r.vanlingen@derma.umcn.nl entiation of keratinocytes, which makes DPPIV (expression and enzyme activity) a marker standing on its own.

Keywords Psoriasis - Dipeptidyl peptidase IV · Keratin $16 \cdot \mathrm{Ki}-67 \cdot \mathrm{CD} 26$

\section{Introduction}

Psoriasis is considered to be an organ-specific autoimmune disease, caused by a combination of genetic and environmental factors and is triggered and maintained by an activated cellular immune system. Its pathology is mainly characterized by cutaneous inflammatory influx of leukocytes and hyperproliferation and aberrant differentiation of keratinocytes. Within the psoriatic lesion, there is a mutual interaction between these keratinocytes and mononuclear leukocytes by means of a third involved party, the cytokines $[5,11]$. The chemical modification of cytokines and hence the modification of their physiological function is of major importance to understand their actual role in the pathophysiology of the psoriatic process.

Dipeptidyl peptidase IV (CD26) is a $110-\mathrm{kDa}$ membrane-anchored glycoprotein with a variety of functions [1, 4]. In general, it is widely distributed throughout the human body and varies in density and form between various organs and cell types. Its proteolytic properties enable it to directly modify substrates such as cytokines in their natural in vivo form, (e.g., CXCL12, CCL5, CXCL11, CXCL10, GLP-1/2) [1, 4, 7, 14].

In human skin, DPPIV is known to be expressed on keratinocytes and known to be upregulated in psoriasis [4, 13, 19, 22]. However, little data are available concerning the potential functional implications of such an upregulation. Selective blockers of DPPIV activity are currently 
being developed as modulators of immunological responses [15]. Recently, it was shown that such an inhibition could suppress keratinocyte proliferation in vitro and partially restore keratinocyte differentiation in vivo [19]. Mapping the DPPIV glycoprotein in psoriasis may therefore add to our current knowledge. Considering the common grounds of the three main pathological components of psoriasis ( $\mathrm{T}$ cells, keratinocytes and cytokines) and the various properties of DPPIV, it is attractive to speculate that DPPIV might be involved in the modulation of the inflammatory process and the keratinocyte abnormalities in psoriasis.

Ensued from this, our goal was to map the epidermal expression and enzyme activity of DPPIV in relation to known markers of proliferation (Ki-67) and abnormal maturation (Keratin 16) of psoriatic keratinocytes in the epidermis [10]. Coherence in dynamics of expression of these three markers could result in expanding knowledge on the existing epidermal disbalance in psoriasis. We studied the expression and enzyme activity of DPPIV in the dynamic margin zone of the psoriatic lesion in order to investigate whether DPPIV distribution differs with the distribution of $\mathrm{K} 16$ and $\mathrm{Ki}-67$ in clinically uninvolved psoriatic skin, in the early lesion and subsequently in the chronic psoriatic lesion. Accordingly, coherence in dynamics of DPPIV (expression and enzyme activity) with keratinocyte proliferation and differentiation markers during the development of a psoriatic plaque could reveal more insight into the mechanisms behind the known upregulation of DPPIV in psoriatic skin.

\section{Patients and methods}

Patients

A total of seven psoriatic patients (mean age \pm SEM, $63 \pm 3$ years) with mild-to-moderate plaque psoriasis [Psoriasis Area and Severity Index $(\mathrm{PASI}) \geq 2$ and $\leq 12$ ] and a control group, composed of three healthy volunteers (mean age $52 \pm 6$ years), participated in this study. Patients had stable plaque-type psoriasis. None of the participants had used any systemic anti-psoriatic or immune-modulating therapy for at least 4 weeks and no topical therapy for at least 2 weeks prior to the biopsy. The healthy volunteers had no history of dermatological or immunological disease. All participants had given written informed consent before enrollment.

\section{Biopsies}

From the psoriatic patients three 4-mm punch biopsies were obtained, respectively, from the centre of the plaque, the inner margin of the plaque comprising the outmost clini- cally involved area and the clinically uninvolved skin (at least $2 \mathrm{~cm}$ from the lesion). From the healthy volunteers one 4-mm punch biopsy was obtained. All participants received local anesthesia ( $1 \%$ xylocaine/adrenaline) before the biopsies were taken. Skin defects were optionally closed with one suture. The obtained specimens were embedded in Tissue-Tek OCT compound (Sakura, Zoeterwoude, Netherlands), snap-frozen in liquid nitrogen and stored at $-80^{\circ} \mathrm{C}$ until use.

Histochemical methods

\section{Immunohistochemical procedure}

The immunohistochemical staining was performed as described previously [2, 23]. Briefly, cryostat sections of $7 \mu \mathrm{m}$ were sliced onto (3-amino propyl)triethoxysilane (AAS)-coated slides, air-dried for $10 \mathrm{~min}$ and fixed in cold acetone for $10 \mathrm{~min}$. After blocking for endogenous peroxidase for $20 \mathrm{~min}$, sections were incubated with either CD26 monoclonal antibody (1:50) (clone BA-5, Santa Cruz Biotechnology, Santa Cruz, FL), Ki-67 (1:25) (clone MIB-1, DAKO, Copenhagen, Denmark) or anti-Keratin-16 (1:20) (clone LL025, Monosan, Uden, Netherlands), diluted in $1 \%$ BSA/PBS for $1 \mathrm{~h}$. Subsequently, sections underwent secondary incubation with IgG-labelled polymer, horseradish peroxidase antimouse-antibody EnVision+ (DAKO, Copenhagen, Denmark) for $30 \mathrm{~min}$. As a coloring agent aminoethylcarbazole + high-sensitivity substrate chromogen (DAKO, Copenhagen, Denmark) was used for $10 \mathrm{~min}$ at $37^{\circ} \mathrm{C}$. Before and after each incubation, sections were washed with PBS for $15 \mathrm{~min}$. All incubations were performed in a dark, humid environment. Counterstaining was performed with Mayer's hematoxylin. Subsequently, the sections were mounted in glycerol gelatin.

\section{Enzymehistochemical procedure}

The demonstration of DPPIV activity was achieved using the method, described by Khalaf et al. [9] with some minor modifications [22]. The frozen biopsies were sliced into 10$\mu \mathrm{m}$ sections on AAS-coated slides. These were air-dried for $20 \mathrm{~min}$ and fixed in cold acetone for $10 \mathrm{~min}$. For the incubation mixture, $3 \mathrm{mg}$ of glycyl-propyl-4-methoxy-2-naphthylamide (GPMN) (Bachem, Bubendorf, Switzerland) was dissolved in $0.2 \mathrm{ml}$ dimethylformamide. This substrate was added to $4.6 \mathrm{ml}$ of PBS and subsequently mixed with $5 \mathrm{mg}$ of fast blue B salt (FBB) (Sigma-Alderich, St. Louis, MO) dissolved in $0.2 \mathrm{ml}$ dimethylformamide and filtered. The samples were incubated in a humid, dark chamber at room temperature for $45 \mathrm{~min}$ with $200 \mu \mathrm{l}$ of the incubation mixture added to each section. After incubation the slides were washed with distilled water, briefly counterstained with 
Mayer's hematoxylin and immersed for $30 \mathrm{~s}$ in alkaline tap water. After rinsing with distilled water, they were mounted in glycerol gelatin.

\section{Negative and positive controls}

In the immunohistochemical procedure, as a negative control the primary antibody was omitted. As a negative control in the enzymehistochemical procedure, inhibition of DPPIV activity was accomplished by pretreating the slides with $200 \mu \mathrm{l}$ of $5 \mathrm{mM}$ Diprotin A (Peptides International, Louisville, KY) for $15 \mathrm{~min}$ at $37^{\circ} \mathrm{C} \mathrm{[3].} \mathrm{As} \mathrm{positive} \mathrm{control,}$ murine kidney tissue was used in the enzymehistochemical procedure [18, 21].

Image analysis

\section{Quantification}

The immunohistochemical and enzymehistochemical DPPIV-stained sections were analyzed using transmission light microscopy with an objective magnification of $200 \times$. Per patient, three of the different biopsy sections (center, inner margin, clinically uninvolved psoriatic skin) were analyzed for the full width of the section. For quantification of the epidermal DPPIV expression and enzyme activity, the following semi-quantitative five-point scale was used: $0=$ no staining, $1=$ slight staining, $2=$ moderate staining, $3=$ moderately pronounced staining, $4=$ pronounced staining.

In order to analyze $\mathrm{K} 16$ and $\mathrm{Ki}-67$ positive cells, digital photographs were made at $100 \times$ magnification. Each photograph was analyzed using IP-lab software (Scanalytics, USA). For quantification of the number of cells positive for $\mathrm{Ki}-67$ and the percentage epidermal area positive for K16, a defined window was set in the epidermis as the region of interest (ROI) which was representative for the whole section. The total ROI and the K16-positive area within the ROI were measured. The K16 value was expressed as percentage K16-positive area of the ROI (\%). The number of Ki-67 positive cells was determined by counting the Ki-67 positive nuclei in a representative epidermal ROI. A line, following the stratum basale of the ROI, was set out and measured. The number of Ki-67 positive nuclei was expressed as positive cells per mm length of basement membrane.

\section{Statistical analysis}

Results are presented as means $\pm \mathrm{SD}$ of the mean. All analyses were carried out using Statistica ${ }^{\circledR}$ statistical software, version 7.0. Comparison of the non-parametric immunohistochemical and enzymehistochemical DPPIV values for the plaque centre, inner margin and clinically uninvolved skin was performed using the Kruskal-Wallis analysis of variance. For analysis of the markers K16 and Ki-67 concerning the plaque centre, inner margin and clinically uninvolved skin we performed analysis of variance (ANOVA) and if significant, Duncan's post hoc comparison was performed. The Student's $t$ test was carried out to study the difference in expression of markers between the uninvolved psoriatic skin and the healthy volunteers skin. Correlation was tested with the Spearman's correlation test. Statistical significance was set at $P<0.05$.

\section{Results}

Patients

Histologically, the skin of the healthy volunteers was normal (hematoxylin-eosin staining). Seven patients (5 males, 2 females) diagnosed with psoriasis participated. In order to assess the severity of the psoriatic lesion used for biopsy, SUM scores (0-12) were determined. SUM score is a psoriatic lesion severity score, comprising erythema (0-4), induration (0-4) and desquamation (0-4) wherein a score of 0 represents no psoriasis and 12 the highest possible severity of the plaque. The mean SUM score in this study was $5.57 \pm 0.34$.

Epidermal distribution of dipeptidyl-peptidase IV, Ki-67 and Keratin-16 (Fig. 1)

In correspondence with earlier findings [22], upregulation of DPPIV on keratinocytes was seen in all of the patients, although to a different extent. In line with these earlier findings, the immunohistochemical and the enzymehistochemical staining of DPPIV followed a similar epidermal pattern; focally pronounced patches of staining, mainly displaying a honeycomb like structure, in psoriatic sections. In those of the healthy volunteers this pattern was practically absent. In brief, the psoriatic pattern was generally more extensive and predominantly located para-papillary with an optimum in the epidermal area around the top of the papillae. Usually the staining was confined to the lower two third of the total epidermis, or more specifically to the lower three quarters of the stratum spinosum (Fig. 1). In both healthy and psoriatic skin, no staining in the stratum lucidum was observed. In the psoriatic coupes, additional column-like structures could regularly be observed.

The DPPIV immunohistochemical expression and enzyme activity in the centre of the plaque, the inner margin and the clinically uninvolved skin showed a comparable pattern with correlations, respectively, of $0.79,0.85$ and $0.87(P<0.02)$. Considering this comparable pattern, the 
Fig. 1 Expression of DPPIV protein expression, enzyme activity, Keratin 16 and Ki-67 throughout the margin zone of a psoriatic lesion compared to normal skin. Plaque centre, inner margin, clinically uninvolved skin (UIS): $\times 200$ magnification. Healthy volunteers skin (HV): $\times 100$ magnification. Noticeably, in the skin of healthy volunteers the DPPIV enzymeactivity staining creates a brownish background staining

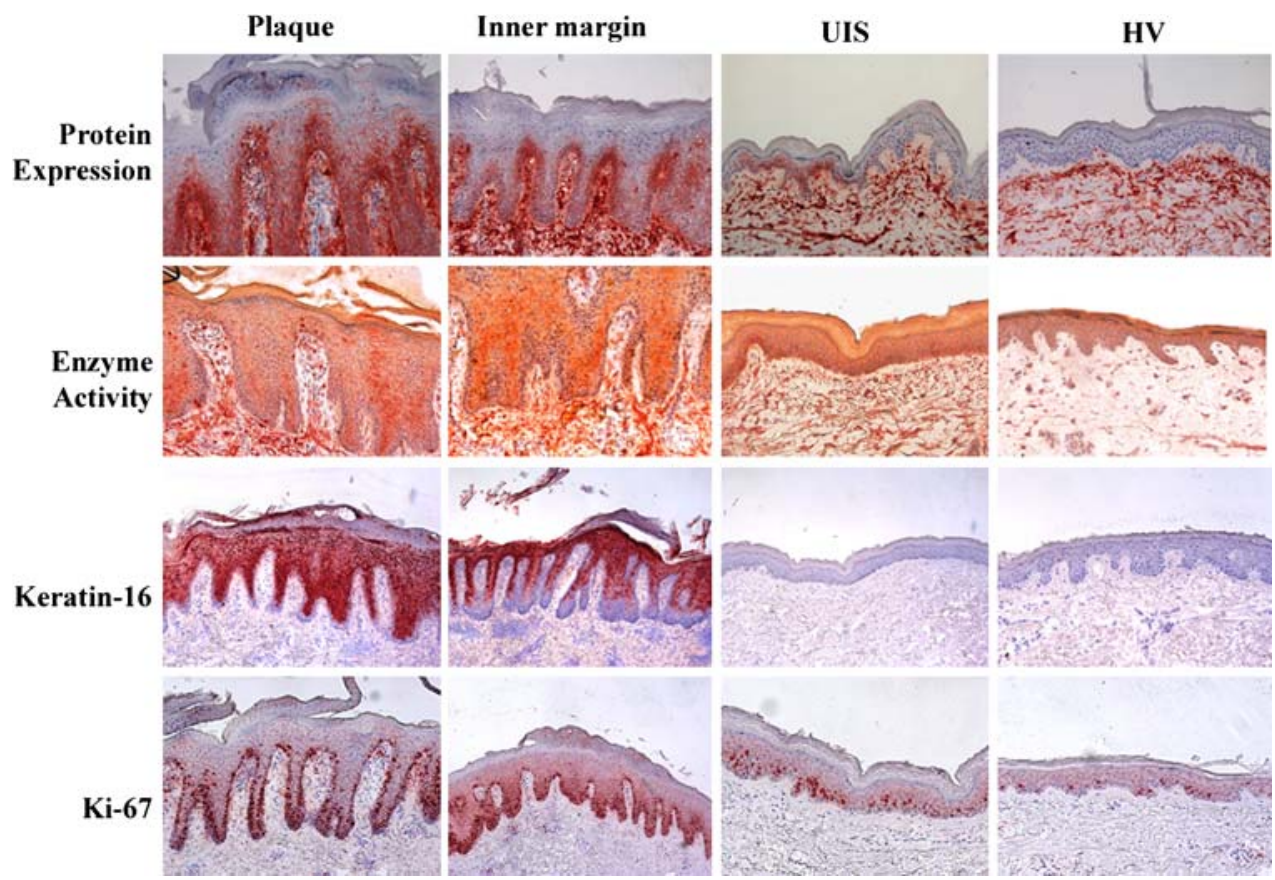

enzyme activity of DPPIV was chosen in this study as a reference for drawing comparisons with the Ki-67 and K16 markers as it encompasses more potential to study dynamics than the protein expression does. Namely, protein expression only confirms the presence of DPPIV whereas enzyme activity denotes presence and activity of DPPIV.

As far as the proliferation marker Ki-67 is concerned, it predominantly appeared in the stratum basale in both psoriatic and healthy skin although to a different extent (Fig. 1). Solitary Ki-67 positive cells could be observed sporadically in the stratum spinosum of the clinically uninvolved skin of psoriatics and the skin of healthy volunteers. Obviously, in the centre and inner margin of the psoriatic lesion, generally a greater number of Ki-67-positive cells was observed in all patients.

Keratin-16, a differentiation marker, only stained positive in the epidermis of the plaque centre and inner margin confirming the aberrant differentiation in these parts as opposed to the clinically uninvolved psoriatic and healthy skin (Fig. 1). The staining was seen throughout the whole stratum spinosum, with an optimum towards the stratum basale.

Dynamics in epidermal distribution of dipeptidyl-peptidase IV, Ki-67 and Keratin-16 (Fig. 2)

Throughout the dynamics of the margin zone of the psoriatic lesion, a stronger significant DPPIV enzymatic activity could be noticed in both the inner margin and centre of the lesion compared to the clinically uninvolved psoriatic skin $(1.38 \pm 0.24$ and $1.57 \pm 0.27$ vs. $0.62 \pm 0.15 ; P<0.01$ and $P<0.05$ ) (Fig. 2). Clinically uninvolved psoriatic skin also showed a significant increase in enzymatic activity compared to skin of healthy volunteers $(P<0.05)$. However, when observing the expression in the centre and the inner margin mutually, no significant difference in expression was detected.

Equal to the DPPIV enzyme activity, Ki-67 antigen was significantly strongly expressed in both the plaque centre
Fig. 2 Mean scores of DPPIV enzyme activity, Ki67 and K16 expression throughout the margin zone. $*=$ significant. (Mean \pm SEM)
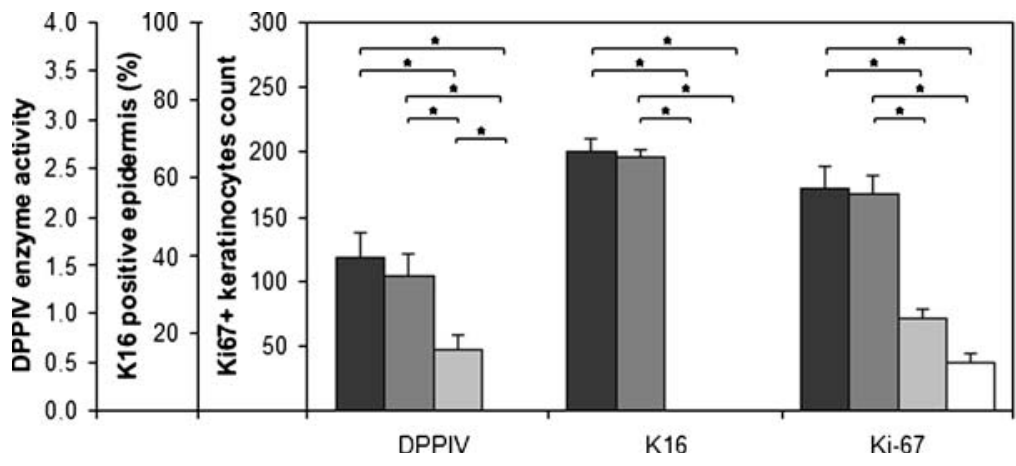

- Plaque Centre $\square$ Plaque Margin 口Uninvolved Skin $\square$ Healthy Volunteer 
and the inner margin compared to the clinically uninvolved psoriatic skin $(172.65 \pm 16.06$ and $167.60 \pm 13.84$ vs. $70.44 \pm 7.97$; both $P<0.01$ ) (Fig. 2). The clinically uninvolved psoriatic skin showed a stronger proliferation tendency of keratinocytes than the healthy volunteers skin, albeit borderline significant $(P=0.057)$. However, when observing the Ki-67 expression in the centre and the inner margin mutually again, no significant difference in expression was detected.

With respect to the expression of K16 in the various parts of the margin zone, the plaque centre and the inner margin showed significantly more expression of K16 in comparison to the clinically uninvolved psoriatic skin $(67.0 \pm 3.13$ and $65.43 \pm 2.1$ vs. $0 \pm 0)$ (Fig. 2). This was in line with the expected expression pattern because in uninvolved psoriatic skin and healthy volunteers skin there exists a natural absence of K16 expression. Mutually however, the centre and margin did, once more, not differ significantly in K16 expression.

Correlation of epidermal distribution of dipeptidyl-peptidase IV, Ki-67antigen and K16

In general, there were no significant correlations observed between enzymatic cytochemical activity of DPPIV and expression of aberrant proliferation or differentiation of keratinocytes throughout the dynamics of the margin zone.

Remarkably, the Ki-67 positive cell count and percentage of K16 positively stained epidermis showed a strong overall correlation in the psoriatic patients, taking together both the plaque centre and the inner margin $(\sigma=0.88$; $P<0.05)$. Though, when investigating the correlation of these markers per locus separately, no significant correlation could be observed (plaque centre $\sigma=0.54$; inner mar$\operatorname{gin} \sigma=0.3$ ).

\section{Discussion}

Dipeptidyl peptidase IV has diverse properties as an ectopeptidase, enabling it to play a key role in the chronic psoriatic immune response which is mainly orchestrated by $\mathrm{T}$ cells, cytokines and keratinocytes in psoriasis. Direct evidence that DPPIV inhibitors are relevant to keratinocyte biology has been provided by the observation that such inhibitors can suppress keratinocyte proliferation in vitro and can partially restore keratinocyte differentiation in vivo [19]. This would imply that DPPIV may affect and interact with the aberrant keratinocyte abnormalities in psoriasis. In line with previous findings [22], the present study confirms a strong upregulation of DPPIV on keratinocytes in psoriatic epidermis but additionally reveals it to be continuously present throughout the dynamics of the margin zone. Moreover, it aimed to shed light on the functional implication of this upregulation combining it with the expression of markers characteristic for proliferation (Ki-67) and differentiation (K16) in psoriatic keratinocytes as coherence in dynamics of expression of these markers could result in creating an epidermal biomarker in psoriasis.

Topographically, the distribution of the enzyme activity of DPPIV did not coincide entirely with the areas of hyperproliferation and aberrant differentiation of keratinocytes in a psoriatic section which ultimately makes DPPIV expression (enzyme activity and protein expression) an entity in itself in psoriasis. The margin zone was previously proven to be a suitable model for studying the dynamics of a psoriatic lesion by representing the chronic and the early process and the uninvolved skin $[6,20,23]$. The inner margin was presumed to represent an early active lesion and therefore reflecting a different level of expression. DPPIV enzyme activity, Ki-67 and K16, all three show, throughout the margin zone, significant upregulation in the centre and inner margin of the plaque compared to the clinically uninvolved skin and the healthy volunteers skin. However, mutually the upregulation in expression in the centre and inner margin did not differ significantly for both the Ki-67, K16 marker as well as for DPPIV enzyme activity. One could suggest that in this study within a psoriatic lesion there exists a homogeneous level of keratinocyte proliferation, differentiation and DPPIV enzyme activity as parts representing an early (inner margin) or more chronic (center) lesion do not seem to make a difference in expression for the markers under study. Moreover, correlation tests applied to the margin zone in this investigation showed no coherence in dynamics of epidermal DPPIV enzyme activity, hyperproliferation and aberrant differentiation of keratinocytes.

The symptomless skin at 2-cm distance from a psoriatic lesion proved to have significantly elevated DPPIV enzyme activity as compared to skin of healthy volunteers, whereas Ki-67 and K16 were essentially slightly present or absent in the symptomless skin. Although the symptomless skin may harbor some "preclinical lesions" or can be subclinically involved, we can conclude that in an early phase of the psoriatic process well before an overt lesion has developed, an increased DPPIV enzyme activity is present clearly showing that DPPIV is involved in an early phase of the psoriatic cascade [8, 12].

A number of other molecules exhibiting DPPIV-like enzyme activity have been introduced recently, and termed "DPP-IV activity and/or structure-homologues" (DASH) [17]. This group comprises several proteases including attractin, DPP 8 and $9[16,17]$. DASH have potential abilities to complement and/or functionally substitute DPPIV on the level of its enzymatic activity. In this light, a marginal note 
has to be made as the DASH might be involved in DPPIVlike processes (as could be the case in the current investigation). Due to the ubiquitous expression pattern and multifunctional nature of DPPIV specifically and the majority of the DASH in general, it may be difficult to deduce the selectivity of DPPIV from the DASH group. However, our results did not show discrepancies between the immunohistochemical and enzymehistochemical staining, which implies that the possible presence of DPPIV homologues does not interfere with the interpretation of the data in the current study.

Our goal was to determine whether the distribution of DPPIV in the epidermis throughout the dynamics of the margin zone, coheres with markers for proliferation and differentiation of keratinocytes in psoriasis in order to expand knowledge on the existing epidermal dysbalance in psoriasis. Considering the localization of DPPIV expression and enzyme activity in the epidermis, being primarily concentrated towards the (supra) basal layers, it is possible to presume an active role for DPPIV in the proliferation of keratinocytes in the pathogenesis of psoriasis. On the other hand, considering the functional enzymatic characteristics of DPPIV, it may well be feasible that the peptidase activity actually restrains the inflammatory disbalance in the psoriatic skin through down-regulation of cytokines secondarily affecting the keratinocyte malfunctioning. Therefore, the psoriatic skin represents an interesting effector organ for DPPIV inhibitors which could tackle not only keratinocytes and cytokines but also (DPPIV+) T cells, key players in the psoriatic pathogenesis all sharing common grounds with DPPIV. Future studies should address the effect of such inhibitors in psoriasis.

In conclusion, we have demonstrated differential expression and activity of DPPIV in normal and diseased skin throughout the dynamics of the margin zone. In addition, we have shown that DPPIV (protein expression and enzyme activity) is expressed well before the development of the overt psoriatic lesion before the accumulation of $\mathrm{T}$ cells and other inflammatory cells. Moreover, the DPPIV enzyme activity does not increase significantly in the more chronic phase of the psoriatic process. Therefore, DPPIV is a consistent feature of the psoriatic lesion. The abnormal enzyme activity in psoriatic diseased skin does not seem to correlate with known markers of aberrant growth and differentiation of keratinocytes, which makes DPPIV a marker standing on its own. Future studies should focus on whether DPPIV can be regarded a useful biomarker for therapy evaluation.

Open Access This article is distributed under the terms of the Creative Commons Attribution Noncommercial License which permits any noncommercial use, distribution, and reproduction in any medium, provided the original author(s) and source are credited.

\section{References}

1. Boonacker E, Van Noorden CJ (2003) The multifunctional or moonlighting protein CD26/DPPIV. Eur J Cell Biol 82:53-73

2. Bovenschen HJ, Seyger MM, van de Kerkhof PC (2005) Plaque psoriasis versus atopic dermatitis and lichen planus: a comparison for lesional $\mathrm{T}$ cell subsets, epidermal proliferation and differentiation. Br J Dermatol 153:72-78

3. Christopherson KW, Cooper S, Broxmeyer HE (2003) Cell surface peptidase CD26/DPPIV mediates G-CSF mobilization of mouse progenitor cells. Blood 101:4680-4686

4. De Meester I, Korom S, Van Damme J, Scharpe S (1999) CD26, let it cut or cut it down. Immunol Today 20:367-375

5. Gaspari AA (2006) Innate and adaptive immunity and the pathophysiology of psoriasis. J Am Acad Dermatol 54:S67-S80

6. Gerritsen MJ, Elbers ME, de Jong EM, Van de Kerkhof PC (1997) Recruitment of cycling epidermal cells and expression of filaggrin, involucrin and tenascin in the margin of the active psoriatic plaque, in the uninvolved skin of psoriatic patients and in the normal healthy skin. J Dermatol Sci 14:179-188

7. Gorrell MD, Gysbers V, McCaughan GW (2001) CD26: a multifunctional integral membrane and secreted protein of activated lymphocytes. Scand J Immunol 54:249-264

8. Hammar H, Hellerstrom C (1970) The oxygen consumption of the germinal epithelium in normal and psoriatic skin. Br J Dermatol 83:371-375

9. Khalaf MR, Aqel NM, Hayhoe FG (1987) Histochemistry of dipeptidyl aminopeptidase (DAP) II and IV in reactive lymphoid tissues and malignant lymphoma. J Clin Pathol 40:480-485

10. Kuijpers AL, Van Pelt JP, Bergers M, Boegheim PJ, Den Bakker JE, Siegenthaler G, Van de Kerkhof PC, Schalkwijk J (1998) The effects of oral liarozole on epidermal proliferation and differentiation in severe plaque psoriasis are comparable with those of acitretin. Br J Dermatol 139:380-389

11. Lowes MA, Bowcock AM, Krueger JG (2007) Pathogenesis and therapy of psoriasis. Nature 445:866-873

12. Mier PD Cotton DWK (1976) The molecular biology of skin. Blackwell, Oxford

13. Novelli M, Savoia P, Fierro MT, Verrone A, Quaglino P, Bernengo MG (1996) Keratinocytes express dipeptidyl-peptidase IV (CD26) in benign and malignant skin diseases. Br J Dermatol 134:1052-1056

14. Oravecz T, Pall M, Roderiquez G, Gorrell MD, Ditto M, Nguyen NY, Boykins R, Unsworth E, Norcross MA (1997) Regulation of the receptor specificity and function of the chemokine RANTES (regulated on activation, normal $\mathrm{T}$ cell expressed and secreted) by dipeptidyl peptidase IV (CD26)-mediated cleavage. J Exp Med 186:1865-1872

15. Pratley RE, Salsali A (2007) Inhibition of DPP-4: a new therapeutic approach for the treatment of type 2 diabetes. Curr Med Res Opin 23:919-931

16. Schade J, Stephan M, Schmiedl A, Wagner L, Niestroj AJ, Demuth HU, Frerker N, Klemann C, Raber KA, Pabst R, von Hörsten S (2008) Regulation of expression and function of dipeptidyl peptidase 4 (DP4), DP8/9, and DP10 in allergic responses of the lung in rats. J Histochem Cytochem 56:147-155

17. Sedo A, Malik R (2001) Dipeptidyl peptidase IV-like molecules: homologous proteins or homologous activities? Biochim Biophys Acta 1550:107-116

18. Smith RE, Reynolds CJ, Elder EA (1992) The evolution of proteinase substrates with special reference to dipeptidylpeptidase IV. Histochem J 24:637-647

19. Thielitz A, Reinhold D, Vetter R, Bank U, Helmuth M, Hartig R, Wrenger S, Wiswedel I, Lendeckel U, Kahne T, Neubert K, Faust J, Zouboulis CC, Ansorge S, Gollnick H (2007) Inhibitors of 
dipeptidyl peptidase IV and aminopeptidase $\mathrm{N}$ target major pathogenetic steps in acne initiation. J Invest Dermatol 127:1042-1051

20. Van de Kerkhof PC, van Rennes H, de Grood R, Bauer FW, Mier PD (1983) Metabolic changes at the margin of the spreading psoriatic lesion. Br J Dermatol 108:647-652

21. van der Velden V, Wierenga-Wolf AF, Adriaansen-Soeting PW, Overbeek SE, Moller GM, Hoogsteden HC, Versnel MA (1998) Expression of aminopeptidase $\mathrm{N}$ and dipeptidyl peptidase IV in the healthy and asthmatic bronchus. Clin Exp Allergy 28:110-120
22. Van Lingen RG, Van de Kerkhof PCM, Seyger MMB, De Jong EMGJ, van Rens DWA, Poll MKP, Zeeuwen PLJM, Van Erp PEJ (2008) CD26 / DPPIV in psoriatic skin: Upregulation and topographical changes. Br J Dermatol (In Press, online early)

23. Vissers WH, Arndtz CH, Muys L, Van Erp PE, de Jong EM, Van de Kerkhof PC (2004) Memory effector (CD45RO+) and cytotoxic (CD8+) T cells appear early in the margin zone of spreading psoriatic lesions in contrast to cells expressing natural killer receptors, which appear late. Br J Dermatol 150:852-859 